\title{
Oral rehabilitation with dental implants in an unusual oral presentation of frontal linear scleroderma "En Coup de Sabre"
}

\begin{abstract}
Localized scleroderma is a clinically distinct inflammatory disease, primarily affecting the dermis and subcutaneous fat. It is characterized by excessive collagen deposition leading to thickening of the dermis and/or subcutaneous tissues, ultimately leading to a scar-like sclerosis. Oral manifestations, including trigeminal neuropathy, xerostomia, thickened periodontal ligament, blunting of mandibular angles on dental radiographs, microstomia, idiopathic resorption of tooth and bone, pathologic mandibular fracture, and poor oral hygiene, are experienced commonly by patients. Given these many oral manifestations, dental treatment may require the involvement of all members of health care team including the rheumatologist, dermatologist, oral surgeon, endodontist, and periodontist, as well as prosthodontist. Many methods of treating partial or complete edentulous PSS patients have been reported in the literature. These methods include maxillary and mandibular complete dentures, implant-retained fixed partial dentures, (maxilla or mandible) overdentures retained by osseointegrated implants and a removable partial denture. The purpose of this report was to present a clinical case of a woman with frontal linear scleroderma "en coup de sabre" associated with an oral rehabilitation using dental implants and prosthodontics.
\end{abstract}

Keywords: dental implants, dermatosclerosis, morphea, dental prosthesis
Volume 8 Issue I - 2017

\author{
Leonardo Matos Santolim Zanettini,' Paulo \\ Eduardo Pittas do Canto, ${ }^{2}$ Maiara Jochims \\ Fumagalli, ${ }^{3}$ Rog rio Miranda Pagnoncelli,' \\ Waldemar Daudt Polido' \\ 'Department of Oral and Maxillofacial Surgery, Pontifical \\ Catholic University of Rio Grande do Sul (PUCRS), Brazil \\ ${ }^{2}$ Department of Prosthodontics, Contento Specialized Dentistry, \\ Brazil \\ ${ }^{3}$ Department of Oral and Maxillofacial Surgery, Indiana School \\ of Dentistry, USA
}

Correspondence: Leonardo Matos Santolim Zanettini, Student of the Master's Program in Oral and Maxillofacial Surgery, Pontifical Catholic University of Rio Grande do Sul (PUCRS):Av. Ipiranga, 668I, Partenon 90619-900, Porto Alegre, Rio Grande do Sul, Brazil,Tel 555000000000

Email leonardo.zanettini@acad.pucrs.br

Received: August 10,2017 | Published: August 22, 2017

Abbreviations: LSCS, linear scleroderma "en coup de sabre", PSS, progressive systemic sclerosis

\section{Introduction}

Localized scleroderma is a clinically distinct inflammatory disease, primarily affecting the dermis and subcutaneous fat. It is characterized by excessive collagen deposition leading to thickening of the dermis and/or subcutaneous tissues, ultimately leading to a scar-like sclerosis. ${ }^{1-3}$ The etiology of morphea remains elusive. ${ }^{4,5}$ Linear scleroderma "en coup de sabre" (LSCS) is a subtype of localized scleroderma. LSCS, usually developing in the first or second decade of life, presents as band-like sclerotic lesions with more or less marked skin discoloration of the frontoparietal area. Involutionary atrophy of skin, muscle, and even bone may occur. ${ }^{6}$ Neurological and ophthalmological abnormalities are not infrequent in LSCS. ${ }^{6}$ En coup de sabre lesions described in literature are mostly in the frontal region near the midline with vertical orientation. Symptoms and clinical findings in LS of the face show variations. ${ }^{7}$ It is a rare disease, with an incidence that varies between 0.34 and 2.7 cases per 100000 population per year. ${ }^{89}$ It is more common in white women (female to male ratio between $2.4: 1$ and $4.2: 1$ ) and its prevalence is similar in children and adults. ${ }^{9,10}$ Oral manifestations, including trigeminal neuropathy, xerostomia, thickened periodontal ligament, blunting of mandibular angles on dental radiographs, microstomia, idiopathic resorption of tooth and bone, pathologic mandibular fracture, and poor oral hygiene, are experienced commonly by patients. ${ }^{11}$ Given these many oral manifestations, dental treatment may require the involvement of all members of health care team including the rheumatologist, dermatologist, oral surgeon, endodontist, and periodontist, as well as prosthodontist. ${ }^{12}$ Many methods of treating partial or complete edentulous PSS patients have been reported in the literature. These methods include maxillary and mandibular complete dentures, implant-retained fixed partial dentures, (maxilla or mandible) overdentures retained by osseointegrated implants and a removable partial denture. ${ }^{13}$ The purpose of this report was to present a clinical case of a woman with frontal linear scleroderma "en coup de sabre" associated with an oral rehabilitation using dental implants and prosthodontics.

\section{Case presentation}

A 65-year-old patient, was seen at the Oral and maxillofacial surgery service at the private clinic Contento- Specialized Dentistry with functional and esthetic complaints. Intra-oral clinical examination, whole face tomography and facial analysis found the patient to have missing teeth ${ }^{13-16}$ and a facial deformity/scar, present as linear sclerotic lesions with skin discoloration in the frontoparietal area, classified as "Facial Linear Scleroderma En Coup de Sabre" (Figures 1\&2). The risks and benefits of each alternative of dental treatment were evaluated and chosen option recommended to correct the functional and esthetic patient complaints was the placement of short implants in the posterior region, aiming at the restoration with a fixed partial prosthesis. Surgical planning included tomography, facial and models analysis, plus virtual plotting using digital software to simulate the final post-surgical result. The surgical plan defined placement of Straumann SLActive Standard plus $3.3 \mathrm{~mm}$ diameter by $10 \mathrm{~mm}$ length implant in the region of $13,4.1 \mathrm{~mm}$ diameter by $8 \mathrm{~mm}$ height in the regions of teeths 14 , and $4.1 \mathrm{~mm}$ diameter by $6 \mathrm{~mm}$ height in the region of. The restoration plan was perform a fixed partial 
prosthesis with splinted implants. The surgery was performed under local anesthesia. The anesthetic used was Articaine 4\% 1:100.000. It is important that the mouth opening of the patient should allow adequate instrumentation at the posterior region. After the muco-periosteal incision and exposure of the alveolar ridge, perforations were made following the conventional protocol system used (Straumann Standard Plus). The basic requirements for implant placement surgery, such as good primary stability and the correct position guided by future restoration were observed. In the case described, good primary stability was obtained. The patient was followed-up weekly. Antibiotic therapy and analgesia were prescribed, and also control computed tomography was conducted after 2 months (Figures $3 \& 4$ ).
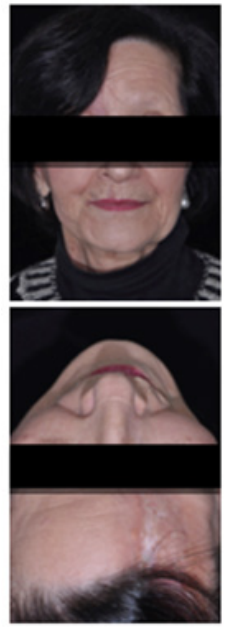

Figure I Clinical aspect of the facial scar. Frontal, sagital and coronal view, present as linear sclerotic lesions with skin discoloration in the fronto-parietal area.

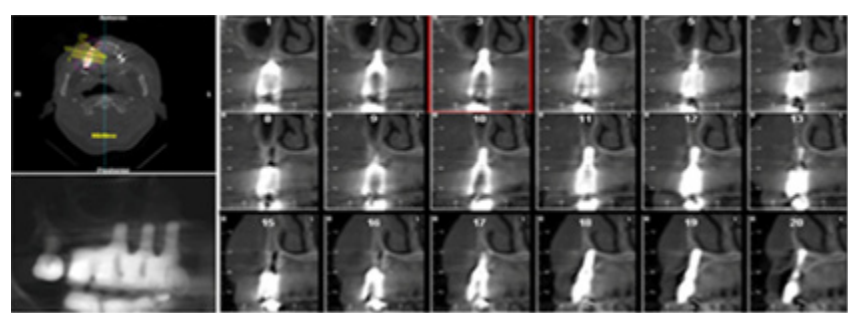

Figure 2 Cone Beam Computed Tomography (CBCT) to evaluate bone density and height to surgical planning.
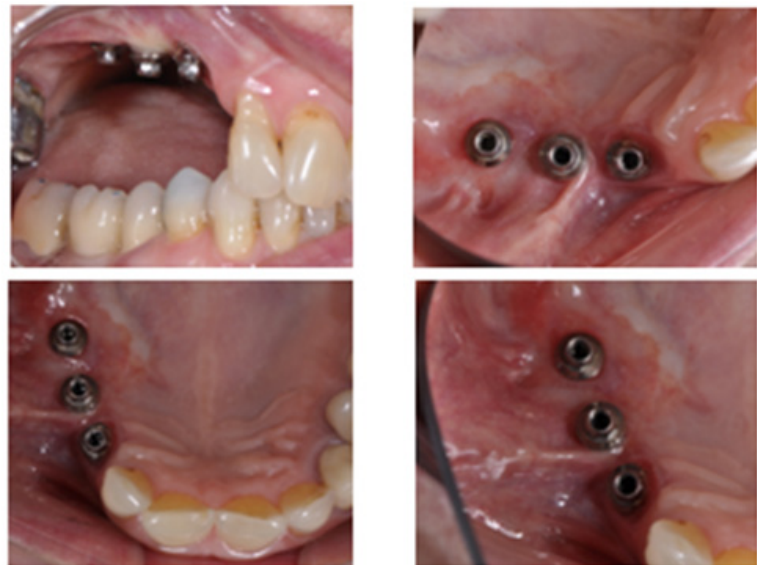

Figure 3 Intra-oral clinical aspect after 24 months of healing. Tissue stability, aesthetic and functional reestablish.
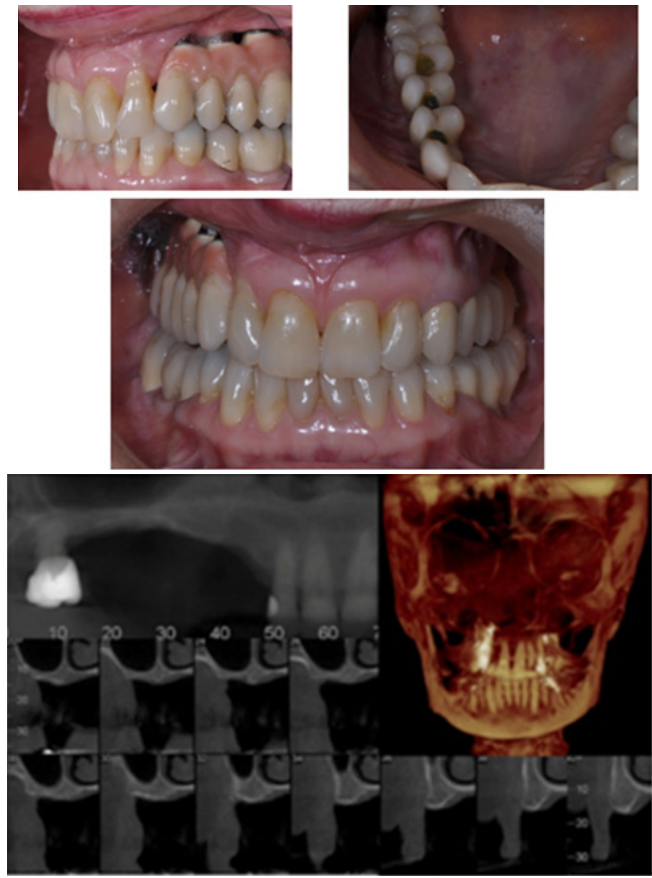

Figure 4 Postoperative CBCT after 24 months for post-operative followup. No pocket formation or other radiographic abnormalities around the implants.

\section{Discussion}

Martin $^{13}$ suggests that Progressive Systemic Sclerosis (PSS) is an autoimmune multisystem rheumatic condition that affects connective tissues of various organs, including Orofacial connective tissue. Many oral manifestations, including xerostomia, microstomia, decreased vascularity, bone resorption, and tooth mobility may affect the choice and survival of dental implants in affected patients. Microstomia, bone resorption, and potential adjacent tissue reaction to implants may be detrimental to the provision of implant technology in edentulous PSS patients. In the patient of this study, there were no signs of xerostomia and the mouth opening was good. The treatment plan was the placement of dental implants and fixed prosthesis, aiming to correct the functional and esthetic patient complaints. Jensen and Sindet-Pedersen 14 reported on a case of the use of osseointegrated implants for reconstruction in a PSS patient with multiple external and internal root resorptions. Four months after the implantation, it was possible to connect abutments on only six out of the nine implants; one implant was not integrated and the two most distal implants were not possible to connect to due to microstomia. At 24 months follow up, the authors reported no pocket formation or other radiographic abnormalities around the implants. It should be noted that the authors strongly stressed the importance of the success in controlling the scleroderma itself, which they thought to be responsible for the favorable biologic reaction to the implants in this patient. Further, the authors stated that even though the implants remained healthy at two years follow up, it was impossible to predict the long term outcome of treatment since reaction to the implants might ultimately develop in the adjacent connective tissue. Pace et al. ${ }^{15}$ reported in 2010 a case of frontal linear scleroderma with intraoral involvement. Although the compromise of the skin was much more aggressive in this case, the intraoral involvement had some peculiarities. The authors reported the presence of a fibrotic lesion extending onto the attached gingiva, associated with a mobile upper right central incisor 
together with marked gingival recession on the mesial aspect of the lateral incisor. Clinical, radiological and histopathological features of other reports of oral involvement in linear scleroderma are very similar to the ones present in our case. ${ }^{16-18}$ Some differences could be seen regarding the extension of and alveolar bone compromise, probably attributed to the progression stage at the moment the diagnosis was made. At present there are only a few cases reports on the provision of osseointegrated implants in a well controlled (treated) PSS patient. It is not possible to predict the survival of the tooth implants given the available information. Other alternatives to treat edentulous PSS patients, who have been reported in the literature, include complete dentures, implant-retained fixed partial dentures, overdentures retained by osseointegrated implants, and a removable partial denture13. However, it should be noted that these reports did not provide information on the prognosis or follow up of these dental procedures. In conclusion, we present a case of linear scleroderma "en coup de sabre" in a female patient. Clinicians should be familiar with the wide spectrum of presentations of scleroderma. As shown in the presented case, the oral rehabilitation can be performed but the surgical planning and risks and benefits should be well evaluated. Its prompt recognition and close follow-up are important to avoid significant local complications

\section{Funding}

None.

\section{Acknowledgments}

None.

\section{Conflicts of interest}

Author declares that there are no conflicts of interest.

\section{References}

1. Tang MM, Bornstein MM, Irla N, et al. Oral Mucosal Morphea: A New Variant. Dermatology. 2012;224(3):215-220.

2. Peterson LS, Nelson AM, Su WPD. Classification of morphea (localized scleroderma). Mayo Clin Proc. 1995;70(11):1068-1076.

3. Mayes MD. Classification and epidemiology of scleroderma. Semin Cutan Med Surg. 1998;17(1):22-26.
4. Fett N, Werth VP. Update on Morphea: I. Epidemiology, clinical presentation and pathogenesis. J Am Acad Dermatol. 2011;64(2):217-228.

5. Laetsch B, Hofer T, Lombriser N, et al. Irradiation-induced morphea: Xrays as triggers of autoimmunity. Dermatology. 2011;223(1):9-12.

6. Gambichler T, Kreuter A, Hoffmann K, et al. Bilateral linear scleroderma "en coup de sabre" associated with facial atrophy and neurological complications. BMC Dermatology. 2001;1:9.

7. Demir Y, Karaaslan T, Aktepe F, et al. Linear Scleroderma En Coup de Sabre of the Cheek. J Oral Maxillofac Surg. 2003;61(9):1091-1094.

8. Silman A, Jannini S, Symmons D, et al. An epidemiological study of scleroderma in the West Midlands. Br J Rheumatol. 1998;27:286-290.

9. Peterson LS, Nelson AM, Su WP, et al. The epidemiology of morphea (localized scleroderma) in Olmsted County 1960-1993. J Rheumatol. 1997;24(1):73-80.

10. Leitenberger JJ, Cayce RL, Haley RW, et al. Distinct autoimmune syndromes in morphea: a review of 245 adult and pediatric cases. Arch Dermatol. 2009;145(5):545-550.

11. Figueiredo MA, Figueiredo JA, Porter S. Root resorption associated with mandibular bone erosion in a patient with scleroderma. Journal of Endodontics. 2008;34(1):102-103.

12. Chaffee NR. CREST syndrome: clinical manifestations and dental management. Journal of Prosthodontics. 1998;7(3):155-160.

13. Martin CW. Provision of dental implants in patients diagnosed with scleroderma. A rapid systematic review. WorkSafeBC Evidence-Based Practice Group. 2009.

14. Jensen J, Sindet-Pedersen S. Osseointegrated implants for prosthetic reconstruction in a patient with scleroderma: report of a case. Journal of Oral \& Maxillofacial Surgery. 1990;48(7):739-741.

15. Pace C, Ward SE, Pace A. A rare case of frontal linear scleroderma (en coup de sabre) with intra-oral and dental involvement. Br Dent J. 2010;208(6):249-250.

16. Van der Veken D, De Haes P, Hauben E, et al. A rare cause of gingival recession: morphea with intra-oral involvement. Oral Surg Oral Med Oral Pathol Oral Radiol. 2015;119(5):e257-e264.

17. Bielsa Marsol B. Update on the classification and treatment of localized scleroderma. Actas Dermosifiliogr. 2013;104(8):654-666.

18. Tang MM, Bornstein MM, Irla N, et al. Oral mucosal morphea: a new variant. Dermatology. 2012;224(3):215-220. 